\title{
On Compton Scattering Scenarios for Blazar Flares
}

\author{
Markus Böttcher ${ }^{1,2} \&$ Charles D. Dermer ${ }^{2}$
}

\begin{abstract}
The synchrotron reflection scenario recently proposed to explain $\gamma$-ray flares observed from blazar jets is studied. Our analysis takes into account the angular distribution of the beamed radiation, the finite extent of the scattering region, and light travel-time effects. We compare energy densities and powers for synchrotron, SSC, reflected synchrotron (RSy), and external Compton (EC) scattering processes. If the width of the scattering layer is much larger than $\Gamma R_{B}^{\prime}$, where $\Gamma$ and $R_{B}^{\prime}$ denote the bulk Lorentz factor and comoving-frame radius of the plasma blob, respectively, then the ratio of the RSy and synchrotron energy densities $\sim 4 \Gamma^{3} n_{\mathrm{BLR}} \sigma_{\mathrm{T}} R_{B}^{\prime}$, where $n_{\mathrm{BLR}}$ is the mean particle density in the broad line region (BLR). Our results imply that Thomson-thick scattering regions of narrow extent must be present for the synchrotron reflection mechanism to operate effectively. This process seems unlikely to cause flares in lineless BL Lac sources, where X-ray and TeV flares are common and the BLR is thought to be weak or absent. We sketch time profiles of flares for various scenarios, including a model where the blob is energized by sweeping up surrounding material.
\end{abstract}

Subject headings: galaxies: active — galaxies: jets — radiation mechanisms: nonthermal

\section{Introduction}

More than 50 blazar-type AGNs have been detected with high confidence by EGRET to emit $\gamma$-rays above $100 \mathrm{MeV}$ (Matox et al. 1997). These sources are identified with flat-spectrum radio sources classified as BL-Lac objects or quasars. Many of these objects exhibit variability on all wavelengths, with some of the most rapid variability, on time scales of hours to days, observed at the highest $\gamma$-ray energies (e.g., Bloom et al. 1997; Wagner et al. 1995; Mukherjee et al. 1997).

The large apparent luminosities in combination with the short variability time scales provide evidence for the widely accepted relativistic jet model for AGNs (for recent reviews, see Schlickeiser 1996 and Hartman et al. 1997), according to which the radio- $\gamma$-ray emission from blazars is emitted via nonthermal synchrotron radiation and Comptonization of soft photons by energetic

\footnotetext{
${ }^{1}$ Department of Space Physics and Astronomy, Rice University, 6100 Main Street, Houston, TX 77005-1892

${ }^{2}$ E. O. Hulburt Center for Space Research, Code 7653, Naval Research Laboratory, Washington, DC 20375-5352
} 
particles in relativistic outflows powered by accreting supermassive black holes. Soft photons which are Compton-scattered to produce the $\gamma$-ray emission include internal synchrotron photons (e.g., Marscher \& Gear 1985, Maraschi et al. 1992, Bloom \& Marscher 1996) and accretion-disk radiation which enters the jet directly (Dermer \& Schlickeiser 1993) and after being scattered by surrounding BLR clouds and circumnuclear debris (e.g., Sikora, Begelman \& Rees 1994; Blandford \& Levinson 1995; Dermer, Sturner, \& Schlickeiser 1997; Protheroe \& Biermann 1997).

It has recently been proposed (Ghisellini \& Madau 1996; hereafter GM96) that the beamed synchrotron radiation, after scattering off a cloud near the jet trajectory and reentering the jet, can be a source of copious soft photons and lead to a pronounced flare of very short duration as the relativistic jet plasma passes through the cloud. Wehrle et al. (1998) argue that this mechanism might explain the February $1996 \gamma$-ray flare observed from 3C 279. A detailed analysis which correctly accounts for causality effects and the finite width of the scattering layer was not performed by GM96, and such a treatement is required before blazar flare spectra and light curves can be modeled.

Here we examine this model in more detail for a simple geometry of the BLR in the limiting regime where the nonthermal jet electron distribution does not evolve. In $\S 2$ we describe the model. Numerical calculations of the magnetic-field and photon energy densities and synchrotron and Compton powers are presented in $\S 3$. Application to blazar flares is made and the effects of blob energization on time profiles of flares are indicated. We summarize in $\S 4$.

\section{Description of the Model}

The idealized system, illustrated in Figure 1, is assumed to have the following qualities: A plasma blob, which in the comoving frame is spherical with radius $R_{B}^{\prime} \sim 10^{15}-10^{17} \mathrm{~cm}$, moves with bulk Lorentz factor $\Gamma \sim 10$ and velocity $\beta_{\Gamma} c$ along the $\hat{z}$ axis which defines the jet. The blob is filled with relativistic, nonthermal electrons and pairs which are isotropically distributed in the comoving frame with a comoving power-law Lorentz factor distribution $n_{e}(\gamma) \propto \gamma^{-p}$ for $\gamma_{1} \leq \gamma \leq \gamma_{2}$. The nonthermal lepton density in the jet is denoted by $n_{\mathrm{e}, \mathrm{jet}}$, and a randomly oriented magnetic field $B$, in equipartition with the energy in relativistic leptons, is present in the blob. An accretion disk is located at $z=0$ and radiates with a luminosity $L_{D} \sim 10^{44}-$

$10^{46} \mathrm{erg} \mathrm{s}^{-1}$. The disk spectrum is approximated by a thermal blackbody of $T_{\text {disk }} \sim 10^{5} \mathrm{~K}$ peaking at the characteristic maximum energy of a standard Shakura-Sunyaev disk spectrum (Shakura \& Sunyaev 1973) for a central black hole of mass $M \sim 10^{8} M_{\odot}$.

The accretion-disk/jet system is assumed to be surrounded by a spherically distributed layer of scattering material which represents the BLR. This layer extends from $r_{\text {in }}$ to $r_{\text {out }}$ and has a radial Thomson depth $\tau_{\mathrm{T}, \mathrm{BLR}} \sim 0.01-0.1$ and a uniform electron density $n_{e}^{\mathrm{BLR}}$. In the following, primed quantities are measured in the reference frame comoving with the relativistic plasma blob, while unprimed quantities are generally referred to the stationary accretion-disk 
frame. For simplicity, we assume that the relativistic electron distribution does not evolve during the outward-motion of the blob. This assumption leads to an overprediction of the contribution from the jet synchrotron mirror for which the accumulation of radiation along the jet trajectory is important. The synchrotron and Compton processes are calculated using $\delta$-function approximations for the emission from relativistic electrons (see, e. g., Dermer et al. 1997).

The important invariant with which to compute the energy density in accretion-disk radiation scattered by the BLR (denoted by the superscript 'ECC' for EC radiation from Clouds) relates the angle-dependent photon number density between the comoving and the stationary frames, given by $n_{\mathrm{ph}}^{\prime}\left(\epsilon^{\prime}, \Omega^{\prime}\right)=\left[\Gamma\left(1+\beta_{\Gamma} \mu^{\prime}\right)\right]^{-2} n_{\mathrm{ph}}(\epsilon, \Omega)$, where $\epsilon=h \nu /\left(m_{e} c^{2}\right)$ is the dimensionless photon energy. Following the treatment outlined by Böttcher \& Dermer (1995), we find that the comoving-frame photon number density at height $z_{2}$ is given by

$$
n_{\mathrm{ph}}^{\prime \mathrm{ECC}}\left(\epsilon_{2}^{\prime} ; z_{2}\right)=\frac{n_{e}^{\mathrm{BLR}} \sigma_{T}}{2 c} \int_{\mu_{2}^{\min }}^{\mu_{2}^{\max }} d \mu_{2} \int_{x_{2}^{\min }}^{x_{2}^{\max }} d x_{2} \frac{\dot{N}_{D}\left(\epsilon_{1}, \Omega_{1}\right)}{r^{2}},
$$

where $\sigma_{T}$ is the Thomson cross section, $\mu_{2}=\cos \alpha_{2}$ (cf. Fig. 1), $\epsilon_{1}=\epsilon_{2}^{\prime} / D_{2}$, and $D_{2}=\Gamma\left(1+\beta_{\Gamma} \mu_{2}\right)$. The term $\dot{N}_{D}\left(\epsilon_{1}, \Omega_{1}\right)$ is the angle-dependent photon production rate of the disk in the direction $\Omega_{1}=\left(\theta_{1}, \phi_{1}\right)$. In the derivation, note that the photon travels opposite to the direction for which $\alpha_{2}$ is defined. The integration limits $\mu_{2}^{\min / \max }$ and $x_{2}^{\min / \max }$ in equation (1) follow from the geometrical constraint that $r_{\text {in }} \leq r \leq r_{\text {out }}$, and from the requirement $x_{s}>R_{B}^{\prime}$, where $x_{s}=x_{2} D_{2}$ is the distance of the location of the scattering event in the BLR to the center of the blob at the time of scattering in the BLR. The latter constraint represents the condition that there is no cold BLR material within the relativistic blob.

The synchrotron reflection mechanism (GM96), where the jet synchrotron radiation is scattered within the BLR and reenters the jet at a later time and at a different location $z_{2}$, is more complicated because light travel-time effects have to be carefully taken into account. The comoving-frame RSy photon number density at height $z_{2}$ is given by the expression

$$
n_{\mathrm{ph}}^{\prime \mathrm{RSy}}\left(\epsilon_{2}^{\prime} ; z_{2}\right)=\frac{n_{e}^{\mathrm{BLR}} \sigma_{T}}{2 c} \int_{\mu_{2}^{\min }}^{\mu_{2}^{\max }} d \mu_{2} \int_{x_{2}^{\min }}^{x_{2}^{\max }} d x_{2} D_{1}^{2} \frac{\dot{N}_{s y}^{\prime}\left(\epsilon_{1}^{\prime}, \Omega_{1}^{\prime} ; z_{1}\right)}{x_{1}^{2}},
$$

where $\epsilon_{1}^{\prime}=\epsilon_{2}^{\prime} /\left(D_{1} D_{2}\right), D_{1}=\left[\Gamma\left(1-\beta_{\Gamma} \mu_{1}\right)\right]^{-1}$, and $\mu_{1}=\cos \alpha_{1} . \quad \dot{N}_{s y}^{\prime}\left(\epsilon_{1}^{\prime}, \Omega_{1}^{\prime} ; z_{1}\right)$ is the angle-dependent synchrotron photon production rate in the comoving frame at height $z_{1}$, where $\Omega_{1}^{\prime}=\left(\alpha_{1}^{\prime}, \phi_{1}^{\prime}\right)$. In the derivation giving equation (2), we use the transformation relating the differential photon production rate between the stationary receiving and comoving frames, given by $\dot{N}_{\mathrm{ph}}\left(\epsilon_{1}, \Omega_{1}\right)=D_{1}^{2} \dot{N}_{\mathrm{ph}}^{\prime}\left(\epsilon_{1}^{\prime}, \Omega_{1}^{\prime}\right)$.

The time of photon emission at location $z_{1}$ is given by the causality condition $z_{1}=z_{2}-\beta_{\Gamma}\left(x_{1}+x_{2}\right)$. Therefore, in addition to the geometrical constraints described in 
the previous paragraph, the condition $z_{1}>0$ has to be met (note that the light travel-time constraint has been ignored in GM96). In particular, causality implies that the photon density due to the RSy process is

$$
n_{\mathrm{ph}}^{\prime \mathrm{RSy}}=0, \text { provided } z_{2}<z_{\mathrm{cr}} \equiv r_{\mathrm{in}} \frac{2 \beta_{\Gamma}}{1+\beta_{\Gamma}} \approx r_{\mathrm{in}}\left(1-\frac{1}{4 \Gamma^{2}}\right) .
$$

For $z_{\text {cr }}<z_{2}<r_{\text {in }}$, only radiation scattered from within a very small angle $\alpha_{1}^{\max } \approx \Gamma^{-1} \sqrt{\left(z_{2} / \beta_{\Gamma} r_{\text {in }}\right)-1}$ contributes to the rescattered photon density in the blob.円 Furthermore, since photons emitted at $z=0$ are at most a distance $\Delta z=\operatorname{ct}\left(1-\beta_{\Gamma}\right) \approx z /\left(2 \Gamma^{2}\right)$ ahead of the blob when it reaches $z$, only a fraction $\Delta z / \Delta r_{\text {BLR }}$ of the radial Thomson depth of an extended cloud can scatter the synchrotron radiation from the blob. As viewed in the comoving frame, this means that unless $r_{\text {out }} \gg \Gamma R_{B}^{\prime}$, no extended photon halo (which contributes to the reflected radiation) will surround the blob as it passes through the BLR.

A relativistically moving blob will receive a considerable flux of scattered synchrotron radiation only when it is passing through the BLR, i.e. for $r_{\text {in }} \lesssim z \lesssim r_{\text {out }}$. Assuming that $\Delta r_{\text {BLR }} \gg \Gamma R_{B}^{\prime}$, we find that the rescattered synchrotron photon energy density can be estimated as

$$
u_{\mathrm{RSy}}^{\prime} \approx 4 \Gamma^{3} \tau_{\mathrm{T}, \mathrm{BLR}} \frac{R_{B}^{\prime}}{\Delta r_{\mathrm{BLR}}}\left(1-\frac{2 \Gamma R_{B}^{\prime}}{z}\right) u_{\mathrm{sy}}^{\prime} .
$$

Two powers of $\Gamma$ come from Thomson scattering, and a third from length contraction and consequent density enhancement of scattering material as viewed in the comoving frame.

In addition to the comoving-frame photon energy densities, $u_{\mathrm{ph}}^{\prime}=m_{e} c^{2} \int_{0}^{\infty} d \epsilon^{\prime} \epsilon^{\prime} n_{\mathrm{ph}}^{\prime}\left(\epsilon^{\prime}\right)$, we compute the total radiative power resulting from Compton scattering of the respective photon fields by calculating the total energy-loss rate of electrons due to Compton scattering. The decline of the Compton scattering cross section in the Klein-Nishina regime, $\epsilon^{\prime} \gamma \gtrsim 1$, is roughly accounted for by neglecting the energy-loss of electrons with Lorentz factor $\gamma$ which scatter photons of energy $\epsilon^{\prime}>1 / \gamma$. The Compton power is

$$
P_{\text {rad }}^{\prime}=V_{B}^{\prime} \frac{4}{3} c \sigma_{T} m_{e} c^{2} \int_{\gamma_{1}}^{\gamma_{2}} d \gamma \gamma^{2} n_{e}(\gamma) \int_{0}^{1 / \gamma} d \epsilon^{\prime} \epsilon^{\prime} n_{\mathrm{ph}}^{\prime}\left(\epsilon^{\prime}\right),
$$

where $V_{B}^{\prime}$ denotes the blob volume. It is important to note that the different Doppler-factor dependences of the observed fluxes from different processes (especially the SSC and EC scattering processes; see Dermer 1995) are properly taken into account.

\footnotetext{
${ }^{1}$ The small solid angle illuminated by the RSy mechanism in comparison with the large solid angle subtended by the BLR clouds in the ECC process could explain why no varying Ly $\alpha$ emission correlated with the highly variable UV continuum was detected from 3C 279 (Koratkar et al. 1998).
} 


\section{Numerical Results and Discussion}

Photon energy densities and radiative powers due to different processes were numerically calculated as a function of the distance of the blob from the accretion disk for a wide range of parameters. Figure 2 shows an example of our series of simulations, using parameters representative of a flat-spectrum radio quasar (FSRQ) and a BL Lac object (BL). Here we let $p=3$ (FSRQ; solid curves) and $p=2.7$ (BL; shaded curves). This choice yields fairly flat $\nu F_{\nu}$ spectra which can be compared with the peaks of the broadband $\nu F_{\nu}$ spectral energy distributions of blazars. The electron density and blob radius are chosen so that the resulting total luminosities are in accord with typical values of the apparent luminosities of FSRQs and BLs, and so that $<100 \mathrm{GeV} \gamma$ rays are not absorbed by $\gamma-\gamma$ pair production on the synchrotron photons intrinsic to the source.

For the generic FSRQ, we assume that the BLR has a radial Thomson depth $\tau_{\mathrm{T}, \mathrm{BLR}}=0.2$ and occupies a spherical shell located between 0.05 and $0.5 \mathrm{pc}$ from the central engine. For simplicity, we assume that the accretion disk radiates isotropically with a luminosity of $10^{46} \mathrm{erg} \mathrm{s}^{-1}$. This choice of parameters gives a Compton power which is $\sim 10$ times greater than the synchrotron power. Figure 2 shows that although the RSy radiation energy density $u_{R S y}^{\prime}$ is larger than $u_{S y}^{\prime}$ when the blob is located within the BLR, the RSy radiative power is about equal to the SSC power. This is because the backscattered jet synchrotron photons are boosted in energy by a factor of $\Gamma^{2}$ relative to the energy of the synchrotron photons in the comoving frame; thus Klein-Nishina effects reduce the radiative power resulting from Compton scattering of RSy radiation more strongly than for the SSC radiation.

In our simulations using plausible parameters compatible with the assumed spherical shell geometry of the BLR, we find that the Compton power due to the synchrotron mirror mechanism is at most comparable to the SSC power. The efficiency of this process is improved when $\Gamma \gg 10$ and a very narrow $\left(\Delta r_{\text {BLR }} \lesssim r_{\text {in }} /\left(2 \Gamma^{2}\right)\right)$, Thomson-thick BLR is located very far $\left(r_{\text {in }} \gg \Gamma^{2} R_{B}^{\prime}\right)$ from the central engine. The synchrotron reflection process might therefore operate in BLR clouds which are thought to surround Seyfert AGNs and FSRQs. BLR clouds, as understood through photoionization models (see, e.g., Wandel 1997 and references therein), consist of dense $\left(n \sim 10^{10}-10^{11} \mathrm{~cm}\right)$, Thomson-thick $(\tau \sim 1-10)$ regions covering a small $(\sim 10 \%)$ fraction of the central engine. For such a model to be feasible, however, the conditions regarding duty cycle and power outlined by Dermer \& Chiang (1998) must be met. We defer presentation of results in the regime $R_{B}^{\prime} \gtrsim \Delta r_{\mathrm{BLR}} / \Gamma$ to future work; the shell geometry used here is excessively artificial in this limit.

The absence of strong emission lines in X-ray selected BLs and (to a lesser extent) radio-selected BLs suggests that the BLR is considerably more dilute in BLs than in FSRQs, and that BLs have mean Thomson thicknesses $\tau_{\mathrm{T}, \mathrm{BLR}} \ll 0.1$ (see Scarpa \& Falomo 1997 and references therein). (On the other hand, the strength of the central ionizing photon source might be much less in BLs than FSRQs.) Superluminal motion observations also indicate that typical values of $\Gamma$ 
for BLs lie in the range between $\sim 3$ and 7 (see the review by Urry \& Padovani 1995). The ability of the synchrotron reflection process to produce gamma-ray flares therefore seems more difficult in BLs than in FSRQs, yet TeV flares often coincident with X-ray flares have been detected from three BL Lac objects (e.g., Punch et al. 1992, Macomb et al. 1995; Catanese et al. 1997, 1998). For the assumed BL parameters in Figure 2, the synchrotron reflection flare could hardly be detected.

If an accretion disk steadily radiates photons (see Böttcher \& Dermer 1995 for a treatment of time-variable disk radiation), then light-travel time effects can be neglected. The accretion disk supplies an abundant supply of soft photons, which can enter the jet directly (ECD) and after being scattered by the BLR (ECC). The comoving photon energy densities from the ECD process can dominate that from the ECC and synchrotron processes when $z \lesssim 10^{-2}$ pc, but declines $\propto z^{-3}$ and $\propto z^{-2}$ farther out (see Dermer \& Schlickeiser 1993; Böttcher, Mause \& Schlickeiser 1997). The ECC photon energy density increases slowly with $z$ when $z<r_{\text {in }}$, and begins to decrease for $r_{\text {in }} \lesssim z \lesssim r_{\text {out }}$. Outside the BLR, when $z \gtrsim r_{\text {out }}$, the energy density asymptotically approaches the limiting behavior $u_{\mathrm{ECC}}^{\prime} \propto z^{-2}$.

When $z \lesssim r_{\text {in }}$, the ECC process dominates over the SSC process provided that

$$
\frac{\tau_{\mathrm{T}, \mathrm{BLR}}}{r_{\mathrm{in}}^{2}} \gtrsim \frac{2}{3} \frac{c B^{2} \tau_{B}}{L_{D} \Gamma^{2}}\left(\frac{p-1}{3-p}\right) \frac{\gamma_{2}^{3-p}-\gamma_{1}^{3-p}}{\gamma_{1}^{1-p}-\gamma_{2}^{1-p}},
$$

where $\tau_{B}=R_{B}^{\prime} n_{\mathrm{e}, \mathrm{jet}} \sigma_{T}$ is the Thomson depth of the blob. Using the parameters adopted in Figure 2 but letting $\tau_{\mathrm{T}, \mathrm{BLR}}, r_{\mathrm{in}}$, and $L$ vary, we find that the ECC photon energy density dominates the synchrotron photon energy density in the comoving blob frame when $r_{\text {in }}(\mathrm{pc}) \lesssim 0.4 \tau_{\mathrm{T}, \mathrm{BLR}}^{1 / 2} L_{46}^{1 / 2}$ and $r_{\mathrm{in}}(\mathrm{pc}) \lesssim 0.1 \tau_{\mathrm{T}, \mathrm{BLR}}^{1 / 2} L_{44}^{1 / 2}$ for the FSRQ and BL parameters, respectively, where $L_{n}=L_{\text {disk }} /\left(10^{n} \mathrm{erg} \mathrm{s}^{-1}\right)$. It should be noted that Klein-Nishina effects are not included in estimate (6).

The bottom panel of Figure 2 illustrates the time profiles of a flare calculated using the FSRQ parameters for the ECC (thick solid curve) and RSy (thick dot-dashed curve) processes. Note that the observer's time element is linearly related to $z$ for a blob moving with constant velocity. The ECC process gives a fast-rise, power-law-decay-type light curve, and the RSy mechanism gives a gradual rise of the $\gamma$-ray flux and a sharp drop as the blob leaves the BLR. A flare produced by the RSy process could be identified by a rapid decline of $\gamma$-rays which is not accompanied by a corresponding decrease of the synchrotron emission. For comparison, we also sketch a flare time profile produced by the ECD process, and time profiles produced by sweeping energization of the blob. In this process, the bulk kinetic energy of the outflowing plasmoid is converted into internal nonthermal particle energy by sweeping up BLR material (see, e.g., Panaitescu \& Mészáros 1998; Dermer \& Chiang 1998; Chiang \& Dermer 1998). The pair of light solid and dot-dashed curves illustrate ECC and RSy flares, respectively, are modeled assuming that the nonthermal electron energy is proportional to the amount of swept-up matter which is then added to a nonthermal lepton distribution accelerated at the base of the jet. Blob deceleration is assumed to be negligible 
here. The upper curves of the two pairs are modeled assuming no radiative losses, and the lower curves of the two pairs illustrate the effects of radiative losses on the nonthermal leptons by crudely multiplying the upper curves by a decaying exponential with a $1.5 \times 10^{18} \mathrm{~cm}$ decay length.

The model RSy light curves, with and without sweeping energization, are similar to $\gamma$-ray light curves observed in the 1991 and 1996 flares of 3C 279 (Hartman et al. 1996; Wehrle et al. 1998). This may be a consequence, however, of the highly idealized BLR geometry used in the calculation. More symmetical flaring profiles observed from PKS 0528+134 (Collmar et al. 1997), PKS 1622-297 (Mattox et al. 1997), and PKS 1406-076 (Wagner et al. 1995) might be more easily explained by the ECD or ECC processes. The declines of the X-ray and optical fluxes correlated with the EGRET $\gamma$-ray fluxes in the February 1996 3C 279 and the 1406-076 flares could, however, rule out the RSy mechanism since the synchrotron component is not directly affected by the reflection process. The inclusion of electron energy evolution and the relaxation of the assumption of a constant velocity blob must be treated to strengthen such conclusions. Future flare modeling must treat the passage of the jet through the BLR clouds, which themselves are in Keplerian motion around the central black hole. The passage of a jet through such a region will display a complicated signature when monitored by different telescopes with different sensitivities and imaging capabilities, which can only be decoded when full account is taken of the processes considered here. The efficiency of the different Compton-scattering scenarios, including the RSy mechanism, in this more realistic system is presently under investigation by the authors.

\section{Summary}

We have analyzed different mechanisms for producing $\gamma$-ray flares which involve relativistic leptons in blazar jets which Compton-scatter soft photons. We presented a detailed study of the jet synchrotron reflection process (Ghisellini \& Madau 1996), where the beamed synchrotron radiation emitted by a relativistic plasma blob is scattered back into the jet by BLR material, and determined conditions under which this mechanism can operate efficiently compared to competing mechanisms. Our calculations show that the efficiency of the synchrotron reflection mechanism is improved for very narrow $\left(\Delta r_{\mathrm{BLR}} \lesssim r_{\mathrm{in}} / 2 \Gamma^{2}\right)$ Thomson-thick $\tau_{\mathrm{BLR}} \gtrsim 0.1$ scattering regions located far $\left(r_{\text {in }} \gtrsim 0.1 \mathrm{pc}\right)$ from the central accretion disk. We argued that the synchrotron reflection model may have serious difficulties explaining $\gamma$-ray flares from BL-Lac objects. Model flare time profiles for various Compton-scattering processes, which in addition include effects of energization of the blob by sweeping up circumnuclear material, were illustrated. The slow-rise, rapid-decay time profile seen in several EGRET flares from FSRQs could be realized by the RSy process, though an accompanying flare from the ECC and ECD processes would also be seen unless the blob only becomes energized after it enters the BLR. A more detailed study of flare production which includes the reflected synchrotron mechanism and sweeping energization in a system where the BLR consists of small, Thomson thick clouds is an important subject for further study. 
MB acknowledges support by the German Academic Exchange Service (DAAD) and by NASA grant NAG 5-4055. The work of CD is supported by the Office of Naval Research and the Compton Gamma Ray Observatory Guest Investigator Program.

\section{REFERENCES}

Blandford, R. D., \& Königl, A. 1979, ApJ, 232, 34

Blandford, R. D., \& Levinson, A. 1995, ApJ, 441, 79

Bloom, S. D., \& Marscher, A. P., 1996, ApJ, 461, 657

Bloom, S. D., Thompson, D. J., Hartman, R. C., \& von Montigny, C., 1997, in 4th Compton Symposium, ed. C. D. Dermer, M. S. Strickman, \& J. D. Kurfess (New York: AIP), 1262

Böttcher, M., \& Dermer, C. D., 1995, A\&A 302, 37

Böttcher, M., Mause, H., \& Schlickeiser, R., A\&A, 324, 395

Catanese, M., et al. 1997, ApJ, 487, L143

Catanese, M., et al. 1998, ApJ, in press

Chiang, J., \& Dermer, C. D. 1998, ApJ, submitted

Collmar, W., et al., 1997, in Proc. of the XXV. ICRC, Vol. 3, 101, ed. M. S. Potgier, B. C. Raubenheimer, \& D. J. van der Walt

Dermer, C. D., 1995, ApJ, 446, L63

Dermer, C. D., \& Chiang, J., 1998, New Astronomy, 3, 157

Dermer, C. D., \& Schlickeiser, R., 1993, ApJ, 416, 458

Dermer, C. D., Sturner, S. J., \& Schlickeiser, R., ApJS, 109, 103

Ghisellini, G., \& Madau, P., 1996, MNRAS 280, 67

Hartman, R. C., et al. 1996, ApJ, 461, 698

Hartman, R. C., Collmar, W., von Montigny, C., \& Dermer, C. D., in The Fourth Compton Symposium, ed. C. D. Dermer, M. S. Strickman, \& J. D. Kurfess (New York: AIP), 307

Hummel, C. A., Krichbaum, T. P., Witzel, A., et al., 1997, A\&A, 324, 857

Koratkar, A., Pian, E., Urry, C. M., \& Pesce, J. E. 1998, ApJ, 492, 173

Macomb, D. J. et al. 1995, ApJ, 449, L99; (e) 1996, 459, L111

Maraschi, L., Ghisellini, G., \& Celotti, A., 1992, ApJ, 397, L5

Marscher, A. P., \& Gear, W. K., 1985, ApJ, 298, 114

Marscher, A. P. 1990, in Parsec-Scale Radio Jets, ed. J. A. Zensus \& T. J. Pearson (New York: Cambridge University Press), 236 
Mattox, J. R., et al., 1997, ApJ, 481, 95

Mukherjee, R., Bertsch, D. L., Bloom, S. D., et al., 1997, ApJ 490, 116

Panaitescu, A., \& Mészáros, P. 1998, ApJ, 492, 683

Protheroe, R. J., \& Biermann, P. L. 1997, Astroparticle Physics, 6, 293

Punch, M., et al., 1992, Nature 358, 477

Scarpa, R., \& Falomo, R. 1997, A\&A, 325, 109

Schlickeiser, R., 1996, Space Sci. Rev., 75, 299

Shakura, N. I., Sunyaev, R. A., 1973, A\&A, 24, 337

Sikora, M., Begelman, M. C., \& Rees, M. J., 1994, ApJ, 421, 153

Steffen, W., et al., A\&A, 302, 335

Urry, C. M., \& Padovani, P. 1995, PASP, 107, 803

Wagner, S. J., et al. 1995, ApJ, 454, L97

Wandel, A. 1997, ApJ, 490, L131

Wehrle, A., et al. 1998, ApJ, in press 


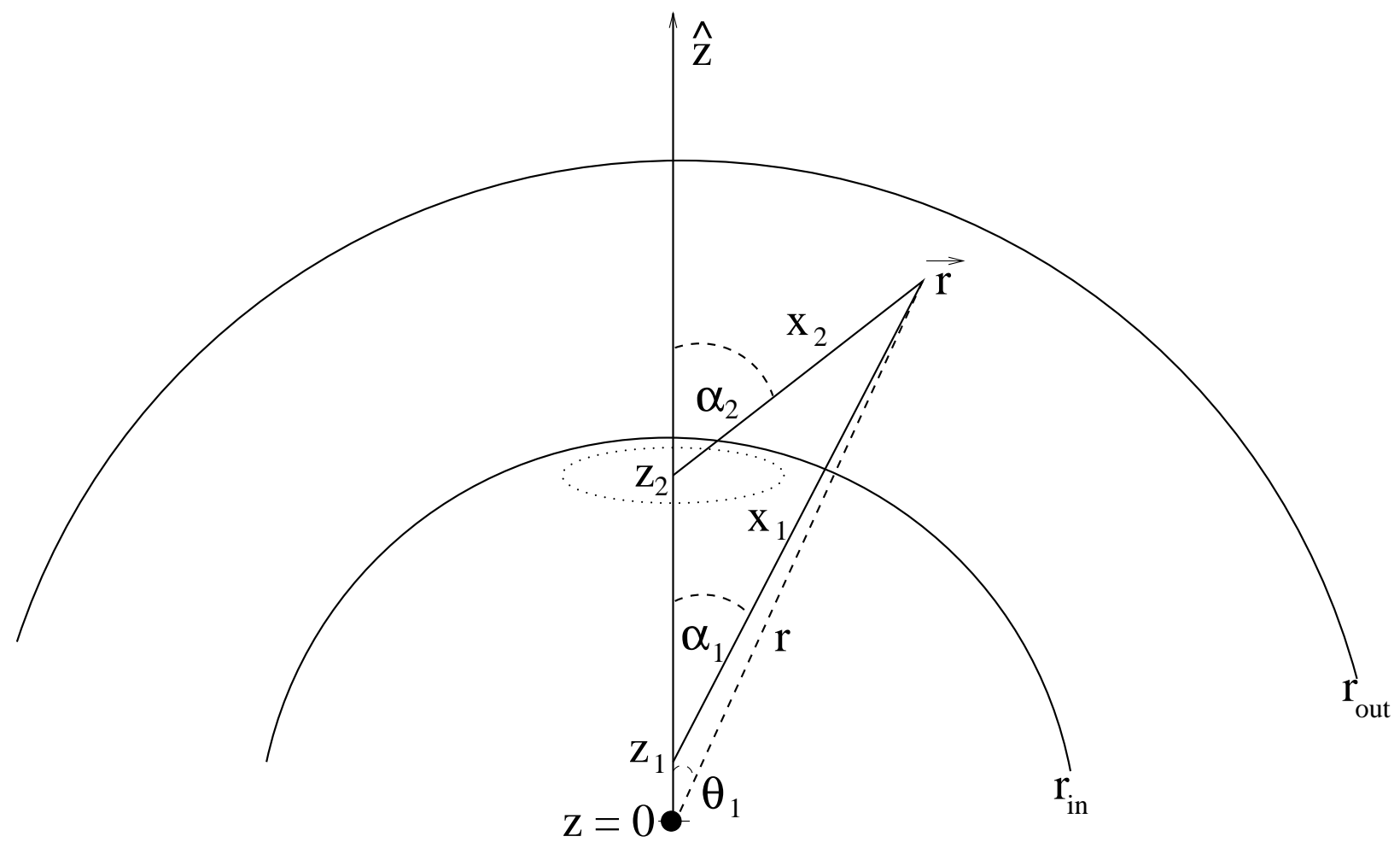

Fig. 1.- Geometry used for the computation of jet radiation rescattered into the jet trajectory. Radiation is emitted at distance $z_{1}$ from the accretion disk at $z=0$, is rescattered in the BLR at a distance $r$ from the central engine, and reenters the jet at location $z_{2}$. 

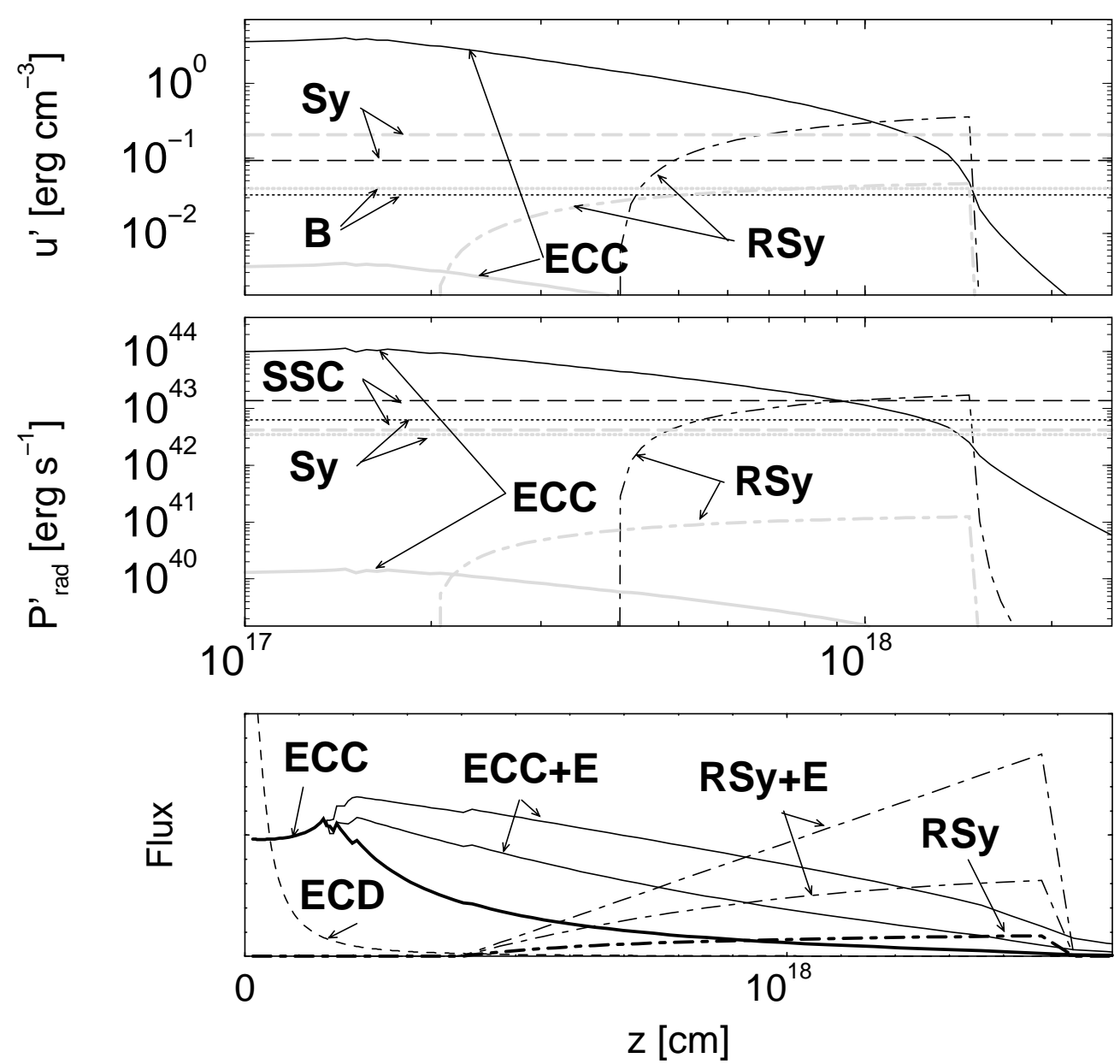

Fig. 2.- Energy densities (upper panel), radiative powers (middle power), and model light cuves (bottom panel) for synchrotron and Compton processes, plotted as a function of distance $z$ of the blob from the central engine. 'ECC' denotes accretion-disk photons scattered from the BLR into the blob, 'RSy' denotes reflected synchrotron photons, 'ECD' denotes accretion-disk photons entering the blob directly, and ' $+\mathrm{E}$ ' curves illustrate the effects of sweeping energization on the blob as it passes through the BLR. Standard parameters used for a typical FSRQ (solid curves) are $\Gamma=10$, $\gamma_{1}=10^{3}, \gamma_{2}=2 \cdot 10^{5}, p=3.0, n_{\mathrm{e}, \mathrm{jet}}=20 \mathrm{~cm}^{-3}, B=0.91 \mathrm{G}, R_{B}^{\prime}=2 \cdot 10^{16} \mathrm{~cm}, r_{\mathrm{in}}=0.05 \mathrm{pc}$, $r_{\text {out }}=0.5 \mathrm{pc}, \tau_{\mathrm{T}, \mathrm{BLR}}=0.2$, and $L_{\text {disk }}=10^{46} \mathrm{erg} \mathrm{s}^{-1}$. Standard parameters used for a typical BL (shaded curves) are the same as for the FSRQ case except that $\gamma_{2}=10^{6}, p=2.7, R_{B}^{\prime}=10^{16} \mathrm{~cm}$, $B=0.99 \mathrm{G}, \tau_{\mathrm{T}, \mathrm{BLR}}=0.02$, and $L_{\mathrm{disk}}=10^{44} \mathrm{erg} \mathrm{s}^{-1}$. Bottom panel uses a linear scale and FSRQ parameters. 